\title{
La importancia de la calidad en el ámbito de la traducción: estudio comparativo entre traductores autónomos, traductores en plantilla y clientes de servicios de traducción
}

\author{
Vanessa Pérez Rodríguez \& Cristina Huertas Abril \\ Universidad de Córdoba \\ z62perov@uco.es \& cristina.huertas@uco.es \\ https://dx.doi.org.10.12795/futhark.2016.ill.06
}

Fecha de recepción: 12.11.2016

Fecha de aceptación: 20.12.2016

Resumen: En este trabajo se presenta un estudio sobre la situación de la calidad y sus normas en el ámbito de la traducción y la localización, así como sobre la situación del uso de las herramientas de evaluación de la calidad en los proyectos de traducción y localización. Para ello, se desarrolla un cuestionario dirigido tanto a traductores profesionales (autónomos o freelance, o que trabajen en empresas o agencias de traducción) como a clientes de este sector. Para el análisis de los datos recogidos mediante el cuestionario se dividen y estructuran los resultados por perfiles; estos son: traductores autónomos o freelance, traductores en empresa o agencia de traducción y clientes del sector. Finalmente, en las conclusiones se muestra el punto de vista de cada perfil sobre la situación de la calidad, sus normas de regulación y las herramientas de evaluación de la calidad, así como una propuesta para estudios futuros.

Palabras clave: calidad, evaluación de la calidad, normas de calidad, traducción, localización.

\section{The importance of Quality in Translation: A Comparative Study between Freelance Translators, In-House Translators and Clients of Translation Services}

\begin{abstract}
In this paper it is presented a study on the situation of the quality and its standards in the field of translation and localization, as well as on the situation of the use of quality evaluation tools in translation and localization projects. To this
\end{abstract}


end, a questionnaire is developed which is addressed to both professional translators (self-employed or freelance, or working for a translation company or agency) and translation clients. For the analysis of the data gathered through the questionnaire, the results are divided and structured by profiles, these being: selfemployed or freelance translators, translators working for a translation company or agency and translation clients. Finally, in the conclusions it is shown the point of view of each profile about the situation of the quality, its standards and the quality evaluation tools, as well as a proposal for further studies.

Key words: quality, quality evaluation, quality standards, translation and localization.

Sumario: I. El concepto de calidad y su aplicación de la traducción yu de la localización. I.I. La normalización: ISO y UNE. I.2. Los sistemas de evaluación de calidad. I.2.I. La evaluación de la traducción como producto. 1.2.2. La evaluación de la traducción como proceso. I.3. Evolución de los sistemas de evaluación de calidad. 2. Metodología. 2.I. Objetivos. 2.2. Descripción de la herramienta de recogida de información. 3. Resultados. 3.I. Traductor autónomo o freelance. 3.2. Traductor en empresa 0 agencia de traducción. 3.3. Cliente de servicios de traducción. 4. Discusión y conclusión.

\section{El concepto de calidad y su aplicación al ámbito de la traducción y de la localización.}

Para entender qué es la calidad y cómo se valora en un proyecto de traducción y localización, debemos empezar definiendo este término. Según el Diccionario de la Lengua Española (DEL), la primera de las acepciones propuestas para esta palabra es "la propiedad o conjunto de propiedades inherentes a algo, que permiten juzgar su valor”. Por lo tanto, la valoración de la calidad conlleva un alto grado de subjetividad, es decir, dos personas juzgando la calidad de un producto o un servicio pueden tener opiniones distintas. Asimismo, y siguiendo con las acepciones propuestas por el DLE, la calidad también es "la adecuación de un producto o servicio a las características especificadas”. Según esta definición deben existir unos patrones o normas que regulen qué productos o servicios tienen la calidad necesaria para distribuirse. Gracias a la creciente internacionalización del mercado desde finales de los años 80 , las empresas son cada vez más conscientes de la importancia de exigir la implantación de unos sistemas específicos que garanticen la calidad de sus productos.

Para conseguir la implantación de dichos sistemas de regulación se han creado diferentes organismos internacionales o nacionales. Uno de ellos, el más conocido, es la organización ISO (International Organization for Standardization en inglés) que, en su sitio web, se define como: 
An independent, non-governmental international organization with a membership of 162 national standards bodies. Through its members, it brings together experts to share knowledge and develop voluntary, consensus-based, market relevant International Standards that support innovation and provide solutions to global challenges. (ISO, 2016)

En esta misma página, ISO define el concepto "standard" o norma como: "International Standards make things work. They give world-class specifications for products, services and systems, to ensure quality, safety and efficiency. They are instrumental in facilitating international trade" (ISO, 2016). Esta definición hace referencia a la calidad como una de las características que deben ser normalizadas y aseguradas. Concretamente, en el campo de la traducción, la organización ISO desarrollará una norma específica para tratar de asegurar la calidad de los proyectos.

Por otro lado, y como hemos mencionado antes, no solo existen organismos internacionales, de hecho, en la definición de ISO se hace mención a “I62 organismos de regulación nacionales". Uno de ellos es AENOR (Asociación Española de Normalización y Certificación) que, en su sitio web, se define como: "Una entidad privada sin fines lucrativos que se creó en 1986. Su actividad contribuye a mejorar la calidad y competitividad de las empresas, sus productos y servicios" (AENOR, 20I0). Así, el término "calidad" aparece en primer lugar como características de regulación imprescindible.

Si consultamos la página reservada para las certificaciones de calidad, AENOR hace referencia a la norma ISO 900 I, de carácter general en la regulación de la calidad; es bajo el epígrafe del sector servicios donde encontraremos la información relativa a la certificación de servicios de traducción. En este sentido, resulta interesante la siguiente reflexión de Pym (2004, p. I5): "Recent years have seen the localization industry pay increasing attention to the importance of translation quality, the lack of which can cost considerable sums in its own right".

Por lo general, una traducción se considera buena o de calidad cuando es lo más fiel posible al texto original $y$, además, está perfectamente redactada en la lengua de llegada, pues a grandes rasgos la finalidad de la traducción y la localización de un producto o servicio es que el consumidor lo sienta como suyo, sin tener que preguntarse de donde es originalmente lo que está consumiendo.

Sin embargo, cuando intentamos ahondar más en la definición de los criterios de calidad que definen una traducción o un producto localizado, es algo más complicado establecer de manera precisa qué se considera de calidad y qué no. Desde el punto de vista lingüístico, por ejemplo, en muchas ocasiones podemos expresar la misma idea de diferentes maneras al traducirlas y todas las versiones pueden ser igual de válidas. La elección de una fórmula u otra se deberá única y 
exclusivamente al criterio subjetivo de quien realiza la traducción, en caso de que el proveedor no nos proporcione glosarios o memorias de traducción para saber qué opciones prefiere para sus traducciones.

Encontramos dos definiciones sobre qué es una traducción de calidad en Defining translation quality (Koby et al., 2014, p. 4). Estas definiciones se presentan desde dos perspectivas diferentes, desde un punto de vista amplio: "A quality translation demonstrates accuracy and fluency required for the audience and purpose and complies with all other specifications negotiated between the requester and provider, taking into account end-user needs". Según esta definición, la calidad se establece únicamente a través del consenso entre cliente y proveedor sobre las especificaciones que se deben cumplir; podríamos asumir que las decisiones en cuanto a aspectos lingüísticos o culturales ocurren dentro de ese consenso entre cliente y proveedor. Por otro lado, encontramos otra definición desde un punto de vista más estricto:

\begin{abstract}
A high-quality translation is one in which the message embodied in the source text is transferred completely into the target text, including denotation, connotation, nuance, and style, and the target text is written in the target language using correct grammar and word order, to produce a culturally appropriate text that, in most cases, reads as if originally writ-ten by a native speaker of the target language for readers in the target culture. (Koby et al., 20I4, p. 4)
\end{abstract}

En este caso, sí se mencionan características más propias de la subjetividad que, como ya hemos comentado anteriormente, dependerán de las decisiones del traductor o localizador en gran medida. Por lo tanto, lo que el consumidor o usuario estará consumiendo, sin ser consciente de ello, será el resultado final de una serie de decisiones o dilemas a los que el traductor o localizador tuvo que enfrentarse en su momento, como afirman Hatim y Mason (1995). Bajo este punto de vista, y siguiendo a estos autores, la traducción se considera desde la perspectiva del producto acabado. Es por esta subjetividad en el producto resultante de la traducción y la localización que debemos diferenciar dos conceptos: el producto y el proceso.

\title{
I.I La normalización: ISO y UNE
}

Hasta 2006, con la publicación de la norma EN-15038, pese a que el sector de la traducción y la localización se encontraba bastante asentado, se carecía de una norma que regulara totalmente el proceso de traducción y localización, desde el mismo momento de la recepción del encargo hasta que llega a las manos del cliente. Sí que existían diversas normativas nacionales e internacionales que se referían sobre todo a la relación entre el traductor y el cliente en cuanto a 
contratos y pedidos, como la norma italiana UNI 10573, las normas austríacas Önorm D 1200 y Önorm D I200, la norma alemana DIN 2345, la norma holandesa Taalmerk o la norma internacional ISO 12616 (Arevalillo, 2004). Como vemos, la norma internacional ISO I26/6, creada en 2002 por la International Organization for Standardization, es la única en esta lista que no regula el servicio y su prestación sino que se centra en la traducción en sí y en el uso terminográfico. En la página web de ISO podemos consultar la definición de esta norma:

The quality of a translation can be measured partly in terms of linguistic elements, such as style and grammar, and partly in terms of the accurate use of the terminology involved. The guidelines in this International Standard provide the necessary elements for quality control of terminological information in translations. The guidelines can also be adapted to provide a basis for the administration of source-language texts, parallel texts, translations, and other information (e.g. bibliographies, references) in the target language. (ISO, 2016)

De acuerdo con esta norma, a través de la regulación de los registros terminográficos, la labor del traductor mejora ya que se sistematiza el proceso de creación de estas bases de datos. Esto permite incrementar la productividad del traductor y la calidad del texto así como facilitar la cooperación entre traductores individuales o equipos de traducción. Para regular cualquier otro proceso general en la prestación de servicios, las empresas deben certificarse en la norma ISO 900I, una de las más importantes y más conocidas. Esta norma es la que establece los sistemas de gestión de calidad de manera general para todo tipo de empresas asegurando una correcta administración de estos sistemas $y$, por consiguiente, un resultado o producto de calidad. Por otro lado, no podemos dejar de mencionar otras normas como la $S A E-J 2450$ que regula la revisión de las traducciones en el sector de la automoción o el LISA QA Model que ofrece un paquete informático para el aseguramiento de la calidad en los procesos de localización. Incluso existen empresas de traducción que utilizan sus propias plantillas de revisión (Arevalillo, 2006).

En vista de la variedad de normas existentes es inevitable pensar en la posibilidad de crear una norma general que englobe todas las demás aunque solo aplicable en el sector de la traducción, al contrario de la norma de calidad ISO, por ejemplo, que es de carácter general y aplicable a cualquier sector. Así nació la norma EN-15038, publicada en 2006. Esta norma reemplazó a las normas nacionales ya existentes hasta el momento en los países miembros del Comité de Normalización Europeo (CEN por sus siglas en inglés), el cual forma parte de los organismos encargados de desarrollar esta norma junto a la Federación Europea de Asociaciones Nacionales de Empresas de Traducción (EUATC por sus siglas en inglés). La finalidad de la nueva norma era establecer unos criterios aceptados por todos los miembros de la EUATC para asegurar un sistema de control de la calidad 
que se llevaran a cabo de manera interna, en cada empresa o agencia, y que fueran aplicables a la gestión de proyectos de traducción. Según Arevalillo (2006: I):

La finalidad de dichos procedimientos, denominados EUATC Quality Standard for Translation Services, consiste en garantizar el cumplimiento de los principios básicos de funcionamiento de las empresas miembros y en evitar así la entrada de empresas o agencias cuyas malas prácticas perjudiquen la imagen de la organización europea y del sector en general.

Más tarde, tras varios debates, se decidió incluir también a los traductores autónomos en la norma.

Nos encontramos ante una norma orientada al proceso de traducción, que cubre los pasos desde la recepción del encargo hasta la entrega final, no es una norma orientada al producto resultante ya que, como se mencionó con anterioridad, cuantificar la calidad de una traducción en sí como producto depende, entre otras cosas, de criterios subjetivos y del consenso al que lleguen el proveedor del servicio y el propio cliente: cuestiones estilísticas, lingüísticas, ortotipográficas, etc. La norma UNE-EN 15038 incluye criterios objetivos y cuantificables consensuados por los comités nacionales que participaron en su creación para conseguir regular y normalizar los procesos desde el interior del sector.

A grandes rasgos, la característica más importante de esta norma es que define el proceso de traducción como una fase más que no tiene valor alguno sin su posterior proceso de corrección y revisión. Para que la traducción se considere de calidad, la corrección y revisión última de la misma debe llevarse a cabo por otra persona distinta al traductor, sin contar la revisión que debe llevar a cabo el autor de la traducción en primera instancia. En este aspecto la norma es flexible ya que no estipula concretamente cómo debe realizarse esa revisión, qué pasos se deben seguir, si no que establece la necesidad absoluta de su realización mediante normas evaluadoras que se integran a la norma general.

Desde el punto de vista de un traductor trabajando en una empresa o agencia, una de las ventajas de esta necesidad de revisión externa, otra persona que no sea el traductor, es que "la responsabilidad de la traducción recae, simplemente, en el proveedor del servicio, que entrega el trabajo al cliente final" (Arevalillo, 2006: 5) y no en el traductor que realiza la traducción. Dada la necesidad de la revisión según esta norma, se entiende que el precio del producto final se incrementará ya que entra un agente más en el proceso: el revisor. Este encarecimiento debería entenderse también como un incremento en la calidad final ya que se está asegurando que la traducción carecerá, en la medida de lo posible, de errores. 
Por otro lado, el sexto capítulo de esta norma habla de los servicios de valor añadido como afirma Arevalillo (2006: 4):

En este capítulo, se mencionan otros tipos de servicios que pueden ofrecerse, además de la traducción en sí, como localización, maquetación, autoedición, gestión de memorias de traducción, glosarios, etcétera. Esto refleja la realidad multidisciplinar de la actividad del traductor y de su capacidad para responder a distintos requerimientos de los clientes.

Estos valores añadidos, por lo tanto, pueden resultar también en un encarecimiento del producto final ya que también están aportando más calidad al mismo. Así, esta es una norma que acepta diversas posibilidades de realización de los procesos, pero siempre enmarcados en una referencia común explicada en su contenido y, como no solo contempla la figura del traductor, trata de establecer las diversas funciones que pueden realizar los diferentes agentes y profesionales a los que da cabida.

Cabe destacar que esta norma ha experimentado cambios durante el año 2015. Si consultamos el sitio web de AENOR, podemos comprobar que en noviembre de este año se publicó la nueva versión de la norma UNE-EN I5038, ahora renombrada como UNE-EN ISO I7I00, y la definición de la misma es la siguiente:

Esta norma internacional proporciona requisitos para los procesos primordiales, recursos y otros aspectos necesarios para la prestación de un servicio de traducción de calidad que satisfaga las especificaciones aplicables.

La aplicación de esta norma internacional ofrece los medios por los que un proveedor de servicios de traducción (PST) puede demostrar la conformidad de los servicios de traducción especificados con esta norma internacional, así como la capacidad de sus procesos y recursos para prestar un servicio de traducción que satisfaga las especificaciones del cliente y otras aplicables. (AENOR, 20I5)

Esta nueva versión de la norma ha mejorado algunos aspectos de la anterior pero lo más importante es que ya forma parte de las normas ISO internacionales. A partir de ahora, las empresas deben usar esta nueva certificación ya que la anterior ha sido reemplazada.

\section{I.2 Los sistemas de evaluación de calidad}

Como ya hemos visto con anterioridad, la valoración de la calidad conlleva un alto grado de subjetividad, de modo que el establecimiento de unos sistemas de evaluación de la calidad válidos para todos los productos y procesos de traducción y localización es una tarea ardua. Asimismo, la calidad suele ser fuente de conflictos entre proveedores y clientes ya que es difícil definir qué es una traducción de calidad. Se deben tener en cuenta muchos factores a la hora de desarrollar un 
proyecto como pueden ser la temática, el vocabulario específico, el destinatario potencial $y$, por supuesto, las preferencias o criterios del cliente, que se pueden evidenciar en forma de glosarios, memorias de traducción, etc. En estos casos, la definición de error puede variar mucho: lo que en un caso puede ser un error grave, en otros, dependiendo del criterio del cliente, por ejemplo, puede ser un error leve o, incluso, puede no ser ni siquiera un error. Todas estas variables influyen en la evaluación final de la calidad y, por consiguiente, en la satisfacción del cliente. Para que los niveles de calidad sean óptimos, de nuevo tenemos que centrarnos en dos aspectos: el proceso y el producto.

\section{I.2.I La evaluación de la traducción como producto}

Para evaluar la calidad de una traducción como producto es importante llevar a cabo una serie de tareas para que esta resulte adecuada. Así, por un lado, una de estas tareas es la revisión que, aunque se considera un proceso dentro de la misma traducción ya que una traducción sin revisión se considera inacabada, este sería el primer paso para depurar errores que podrían deteriorar la calidad del resultado. En definitiva, mediante la revisión, al realizarse sobre un producto que todavía no está acabado, se corrigen los errores de cualquier tipo que se puedan encontrar. Por otro lado, nos encontramos con el control de calidad ( $Q C$ en inglés por Quality Control). La Asociación Española para la Calidad, en su sitio web, define esta tarea de la siguiente manera:

El control de calidad se materializa en las inspecciones y ensayos para comprobar si una determinada materia prima, un semielaborado o un producto terminado, cumple con las especificaciones establecidas previamente.

El control de calidad normalmente se apoya en la estadística para minimizar el número de ensayos a realizar conservando al mismo tiempo la confianza en las conclusiones obtenidas. (AEC, 2016)

En primer lugar, debemos fijarnos en que, en este caso, nos podemos referir a un "producto terminado" (a diferencia de la revisión, que se realizaba sobre un producto todavía en elaboración). En traducción, este control se realiza mediante la lectura y evaluación total y profunda de la traducción terminada pero siempre antes de entregársela al cliente. De este modo, si encontráramos algún error que no se ha detectado en la revisión, se debe corregir o retraducir el fragmento en cuestión dependiendo de la gravedad o extensión del error encontrado. Sin embargo, si lo que queremos es establecer un control de calidad de un proveedor, este se realizaría mediante un muestreo de su producción durante un periodo determinado de tiempo, pero siempre mediante la lectura completa del porcentaje de producción que hayamos considerado.

Otra de las tareas que se deben llevar a cabo para evaluar la calidad de una traducción es el aseguramiento de la calidad, o QA por sus siglas en inglés (Quality 
Assurance). De nuevo, en el sitio web de la Asociación Española para la Calidad podemos ver una definición de esta tarea:

El Aseguramiento de la Calidad consiste, por tanto, en seguir una línea de actuación dirigida a conseguir trabajar en base a un conjunto de acciones planificadas y sistemáticas, implantadas dentro del Sistema de Calidad de la empresa.

Los sistemas de aseguramiento de la calidad tradicionalmente han tenido una gran carga documental puesto que requieren de una planificación exhaustiva, definición de tareas y responsabilidades, registro de resultados obtenidos y pautas de inspecciones internas continuas, todo ello soportado en documentos. (AEC, 20I6)

En este caso, dentro del sector de la traducción, el aseguramiento de la calidad se realiza mediante un muestreo del proyecto que estemos llevando a cabo en el que elegiremos ciertas partes que queramos evaluar en busca de determinado tipo de errores. De este modo, estableceremos si un producto alcanza los niveles adecuados de calidad que se desean.

\section{I.2.2 La evaluación de la traducción como proceso}

Si hablamos de la traducción como proceso, nos encontramos con dos normas que regulan la calidad. Estas normas ya han sido comentadas en el punto 3.2.3 de este trabajo: la norma internacional ISO 12616 que se centra en la traducción en sí y el uso terminográfico, y la norma europea UNE EN-I5038 que reemplaza las anteriores existentes a nivel nacional. Esta es una norma orientada al proceso de traducción, que cubre los pasos desde la recepción del encargo hasta la entrega final. Es importante mencionar de nuevo que, desde finales del año 2015, esta norma ha sido actualizada como norma ISO, de carácter no solo europeo sino mundial.

\section{I.3 Evolución de los sistemas de evaluación de calidad}

Uno de los modelos pioneros en los sistemas de evaluación de la calidad en traducción fue el llamado SICAL (Système Canadien d'appréciation de la Qualité Linguistique), desarrollado en los años 70 por la oficina de traducción del gobierno de Canadá. Según Martínez (2014: 4): "This system aimed at discarding the evaluator's value judgement traditionally dependent on his particular knowledge and appraisal".

Este método fijaba unos parámetros de referencia para comparar las características lingüísticas de traducciones ya terminadas, estableciendo el concepto cuantificable de umbral de aceptabilidad por el cual se aceptaba o se rechazaba una traducción. La práctica de este método establecía que el resultado final resultaba de la división del conjunto de puntos negativos, es decir, los errores, entre las palabras 
del pasaje que se tomaba como referencia para llevar a cabo la medición, generalmente unas 400 palabras. Aunque se puso en duda la efectividad de los resultados y del método (pues el número de errores podía llegar a varios centenares haciendo que la tarea de aplicar este sistema fuese bastante complicada y que el pasaje de 400 palabras escogido podía no ser un buen ejemplo representativo del conjunto del texto traducido), la importancia de este sistema radica en que supuso el punto de partida para el desarrollo de metodologías y normas posteriores.

Hoy en día, la metodología más utilizada para llevar a cabo el aseguramiento de la calidad en el campo de la localización es la norma LISA QA Model. Uno de sus puntos fuertes es que fue desarrollado desde dentro del sector, por profesionales asociados a LISA, $y$, aunque a priori pueda parecer una norma única y exclusivamente aplicable a la localización, sus plantillas son fácilmente adaptables a otros campos de la traducción. Esta norma abarca desde la tarea de la traducción hasta la adaptación de documentación impresa, ayuda en línea e interfaz de usuario. Las plantillas de la norma LISA QA Model fueron desarrolladas y distribuidas por primera vez en 1995 por la asociación LISA. En su segunda versión, en 1999, contaba con competencias mejoradas. La tercera versión vio la luz en 2004 y la última versión, la 3.I, se publicó en 2007, cuatro años antes de la disolución de la asociación.

Mediante esta norma se toma un porcentaje del trabajo, se lee y se examina en busca de errores que serán clasificados según su gravedad. En este caso, se divide la clasificación de errores en tres categorías: errores críticos, errores importantes y errores leves; a su vez, cada categoría de error se puntúa de manera determinada. De este modo, el trabajo se rechazará si este superara un número máximo de puntos en la clasificación de los errores. Así, por ejemplo, en un texto de 50000 palabras se tomaría un porcentaje para evaluar, un $10 \%$ aproximadamente, por lo que se examinarían 5000 palabras. El porcentaje de error máximo por defecto sería del $1 \%$, de manera que este trabajo no se aceptaría si superase 50 puntos de error.

Por otro lado, la norma SAE J2450, de la Society of Automotive Engineers (SAE), está orientada al aseguramiento de la calidad de las traducciones en el sector de la automoción. Es importante llevar a cabo un control de calidad adecuado ya que el lenguaje técnico debe ser específico y carente, en la medida de lo posible, de ambigüedades. Schütz (1999, p. 5) recoge las partes de las que consta esta norma:

The current SAE J2450 metric proposal consists of eight classes ([SAE J2450, 1999]):

I. Wrong term (WT)

2. Omission (OM) 
3. Grammatical error related to word structure, agreement and part of speech (GE) 4. Wrong word order (WO)

5. Misspelling (SP)

6. Punctuation error (PE)

7. Superfluous text (SF)

8. Miscellaneous error (ME)

It is intended that a human classifies these errors into the above eight classes. In a second step she then categorizes the errors into serious (s) and minor $(m)$, and in a third step each error is assigned a weight between one (I) and five (5). 5 corresponds to a very serious error and I indicates an error with a minimum of consequences for the service operation.

La última parte de esta norma consiste en dos metarreglas para desambiguar la asignación de error a las categorías y subcategorías anteriores. Esto es, en caso de ambigüedad en cuanto a la pertenencia del error a una u otra categoría de error, se escogerá la que primero aparezca en la lista. En caso de que la duda sea entre categorizar el error como grave o menor, este se debe establecer como grave. Esta norma fue actualizada por última vez en 2005, versión que se encuentra vigente hoy en día.

En 201I, la Translation Automation User Society (TAUS) desarrolló el Dynamic Quality Evaluation Model (en español: "modelo dinámico de evaluación de calidad”). Esta es la contribución más reciente al sector de la evaluación de la calidad en traducción. Este modelo se considera dinámico ya que, dependiendo del tipo de texto que se traduzca, los requisitos en la medición de la calidad serán diferentes. Se trata de una base de conocimiento muy amplia con recursos y herramientas de acceso libre y gratuito a través de su plataforma web.

\section{Metodología}

\section{I Objetivos}

El principal objetivo de este estudio es evaluar el estado actual de la calidad y las herramientas de control de calidad en el ámbito de la traducción y la localización. Para alcanzar este objetivo principal, se establecen tres objetivos secundarios:

I. Observar si existen diferencias de uso de las herramientas de control de calidad desde la perspectiva del traductor autónomo, y desde la perspectiva de la empresa especializada o agencia de traducción. 
2. Representar la diferencia en la percepción y contrastar la importancia de la calidad desde el punto de vista del traductor profesional, ya sea autónomo o trabajador en plantilla, así como desde la perspectiva del cliente, para observar las posibles diferencias o distorsiones en su percepción.

3. Evaluar la situación actual de aplicación y cumplimiento de las normas para garantizar la calidad, ISO y UNE, en el ejercicio de la traducción y su posible evolución futura, así como establecer, si la hubiese, una conexión entre el uso de las herramientas informáticas de control y evaluación de la calidad, y la aplicación de las normas anteriormente citadas.

\subsection{Descripción de la herramienta de recogida de información}

Para llevar a cabo la recogida de información para elaborar el análisis o evaluación de la calidad y los otros objetivos expuestos anteriormente, se ha optado por desarrollar un cuestionario en línea a través de Google Forms. Consideramos que esta es una herramienta de obtención de datos que, a pesar de sus limitaciones, resulta muy adecuada para nuestro trabajo, pues de acuerdo con Wright (2006):

Current web survey products and services have greatly facilitated the process of creating and conducting online surveys. Researchers can save considerable time by utilizing the products and services that are offered by many of the businesses highlighted in this article, compared to the time that it would take most researchers to create an online survey themselves using a web authoring program, thanks to a variety of attractive features offered.

Para validar el instrumento, los investigadores decidieron aplicar la metodología Delphi (Keeney, Hasson, y McKenna, 2000). La recogida de datos en forma de cuestionario es uno de los métodos más habituales para analizar percepciones y opiniones en una investigación a gran escala (Mackey, y Gass, 2005). Tras este proceso, finalmente el cuestionario se presentó a los participantes del siguiente modo:

- Sección I: Título y presentación del cuestionario.

- Sección 2: Preguntas comunes para traductores profesionales (en plantilla o autónomos) y clientes del sector.

- Sección 3: Preguntas orientadas exclusivamente hacia trabajadores de la traducción sobre el uso de las herramientas informáticas de evaluación y control de calidad, así como sobre la aplicación de las normas reguladoras.

- Sección 4: Agradecimiento y posibilidad de obtener copia de los resultados del estudio a través de correo electrónico. 
El periodo de difusión del cuestionario y recolección de datos tuvo lugar en los meses de junio y julio de 2016.

\section{Resultados}

En el cuestionario que se ha elaborado para realizar el presente estudio sobre la calidad en el ámbito de la traducción y la localización han participado un total de 33 personas, de los cuales 19 son profesionales del sector de la traducción: 10 traductores que trabajan para agencias o empresas de traducción $(31,3 \%$ y 13 traductores autónomos ○ freelance $(40,6 \%)$; los 9 participantes restantes son clientes de servicios de traducción (28,1 \%).

Para llevar a cabo el análisis de los datos, se van a dividir los resultados por perfiles: traductores autónomos o freelance, traductores en empresa o agencia de traducción, y clientes de servicios de traducción, por este orden.

\section{I. Traductor autónomo o freelance}

En primer lugar, las respuestas a la primera pregunta abierta del cuestionario sobre cómo definirían la calidad en el ámbito de la traducción y la localización han sido ambiguas, ya que ha habido participantes que han entendido que la pregunta trataba sobre valorar la calidad en las traducciones. En este caso, la mayoría de las respuestas han sido favorables, solo una de las respuestas ha sido negativa, calificando la calidad como "mediocre"; tres participantes no han aportado respuesta a esta pregunta, a pesar de aparecer marcada como obligatoria en el cuestionario. En el caso de las respuestas completas que han definido el término calidad, encontramos varias que establecen una diferencia entre si la traducción la realiza un profesional o no; de modo que entienden que si la traducción la realiza un profesional será mejor o de mayor calidad.

Las respuestas más relevantes e interesantes para este estudio son las que definen la calidad como uno de los pilares más importantes en la traducción, asociado a aspectos tales como la comprensión y la comunicación del mensaje traducido.

La calidad es el pilar en el ámbito de la traducción; entonces es un principio básico en el que basa la traducción.

Comunicabilidad.

Creo que depende de si contratamos a un profesional o contratamos a cualquier persona que hable varias lenguas y punto.

Entrega puntual, sin errores, idiomático. 
Es la relación entre el producto traducido y cómo sería el texto si originalmente se hubiera escrito en el idioma objetivo; a mayor calidad, menor divergencia.

Esencial.

Importante.

La parte esencial para presentar un buen trabajo.

Tabla I. Respuestas sobre definición de calidad. Traductores autónomos o freelance. Elaboración propia

En cuanto a los problemas a los que se tiene que enfrentar la calidad en el ámbito de la traducción o la localización, para lo cual se ha utilizado una escala Likert ( $I$ = nada importante, 4 = muy importante), el punto de vista de un traductor autónomo o freelance es que los mayores problemas son los requisitos ya que estos son cada vez más exigentes en cuanto a la calidad del producto o el servicio de traducción o localización; 10 de los 13 participantes de este perfil (76,9\%) consideran que este problema es "importante" o "muy importante". La degradación de la calidad se percibe como otro problema relevante, ya que de nuevo el 76,9 \% lo puntúa como "importante" o "muy importante”. Finalmente, la puntuación más baja como problema "importante" la reciben los requisitos de certificación ya que únicamente 5 de los participantes de este perfil $(38,4 \%)$ le dan una puntuación de "importante" o "muy importante", mientras que 7 de ellos $(53,8 \%)$ consideran que se trata de un problema "poco importante".

El siguiente bloque del cuestionario evalúa la importancia de diferentes aspectos durante el proceso de traducción o localización. Entre estos aspectos se encuentra la calidad a la que se le da una importancia muy alta $\left(84,6^{\circ} \%\right)$. El aspecto de la ética u honestidad durante la colaboración entre el profesional del sector y el cliente es otro de los aspectos con mayor puntuación, pues el 61,5\% de los participantes le dan una importancia muy alta. Seguidamente, encontramos los aspectos de rapidez y relación entre cliente y proveedor, ambas características cuentan con $7(53,8 \%)$ y 6 (46,I \%) puntuaciones de máxima importancia, respectivamente. El precio, sin embargo, es un aspecto al que estos profesionales de la traducción otorgan una puntuación de importancia, pero no de la máxima ya que solo el 30,7 \% lo consideran como "muy importante" frente al 53,8 \% como "importante". El aspecto con menor importancia de los reflejados en el cuestionario es el de los procesos que se han de llevar a cabo en cualquier proyecto de traducción o localización, obteniendo 5 puntuaciones de "poco importante" y otras 5 de "importante", el 38,4\% respectivamente, mientras que solo ha obtenido $3(23,07 \%)$ de "muy importante".

Con relación al uso de herramientas informáticas específicas para el control de la calidad, se observa que la mayoría de los participantes $(6 \mathrm{I}, 5 \%)$ sí utiliza alguna de las herramientas disponibles en el mercado actual para llevar a cabo el control de calidad en sus proyectos de traducción o localización, mientras que el $38,4 \%$ reconoce no hacer uso de herramientas informáticas específicas. En este contexto, 
aquellos que han contestado que sí utilizan alguna herramienta informática de control de calidad específica se decantan por el uso de las herramientas integradas en los programas de traducción asistida por ordenador (TAO) como por ejemplo SDL Trados, Memo Q o Déjà Vù (62,5\%). El uso de la herramienta XBench es la siguiente en uso (37,5\%), aunque uno de los tres participantes que ha elegido esta opción ha elegido también el uso de herramientas integradas en programas TAO. Por otro lado, aquellos que reconocen no utilizar herramientas específicas para el control de la calidad han destacado la revisión del trabajo mediante otros compañeros o profesionales, así como el uso de otros materiales para realizar este control.

En el último bloque de preguntas enfocado a la posesión de certificados de calidad, encontramos que ninguno de los participantes de este perfil afirma poseer algún certificado de calidad. Por otro lado, cuando se les pregunta si se plantean obtener alguno en el futuro, 9 de ellos $(69,2 \%)$ responden positivamente, frente a $4(30,8 \%)$ que no lo consideran en el momento del cuestionario.

\subsection{Traductor en empresa o agencia de traducción}

Este perfil es el segundo en cuanto a número de participantes en el cuestionario, (31,3\%). Las respuestas a la pregunta sobre cómo definirían la calidad en el ámbito de la traducción y la localización desde su punto de vista es el que ha proporcionado las respuestas más concretas y exhaustivas de manera general. Como se observa en la siguiente tabla (Tabla 2), estas respuestas hacen hincapié, en la mayoría de los casos, en definir la calidad en traducción como la expresión del texto traducido o el proyecto localizado como propio de la lengua o la cultura meta, si bien también se hace mención a la corrección.

Una traducción de calidad es un texto correcto conceptualmente, expresado en la forma correcta, con corrección lingüística y con un buen formato.

Una traducción de calidad es aquella que parece no haber sido traducida, es decir, que el texto meta se comprende con total naturalidad como si hubiera sido redactado originalmente en ese idioma.

Pese a los avances, siempre se puede mejorar.

La calidad la define el cliente en cada proyecto.

La calidad es el aspecto clave que distingue entre un trabajo realizado por un profesional y uno elaborado por un aficionado. Consiste en prestar atención a las cosas que un lector normalmente pasaría por alto, con el propósito de que el trabajo sea un reflejo fiel al texto original pero adaptado a la cultura de la que formará parte una vez finalizado.

Corrección en muchos aspectos: gramática, puntuación, ortografía, puntualidad, terminología, etc. 
Aspirar a producir un texto que resulte natural y fluido en la lengua de destino, así como cumplir con los requisitos y necesidades del cliente.

Asegurar que el producto final cumple su objetivo.

Tabla 2. Respuestas sobre definición de calidad. Traductores en empresas 0 agencias de traducción. Elaboración

propia.

En la siguiente pregunta, sobre los problemas a los que se tiene que enfrentar la calidad en el ámbito de la traducción y la localización, observamos que el mayor problema para los participantes bajo este perfil es la degradación de la calidad, ya que el $50 \%$ de los participantes le dan una puntuación de "muy importante". El siguiente problema por orden de importancia son los requisitos de calidad cada vez más exigente, con un $40 \%$ y un $30 \%$ de puntuación "muy importante" e "importante", respectivamente. Por último, nuevamente, nos encontramos con los requisitos de certificación como problema relacionado con la calidad al que se le da menor importancia, ya que solo el $20 \%$ le ha otorgado la puntuación de "muy importante".

En el bloque de evaluación de la importancia de diferentes aspectos durante el proceso de traducción o localización, la mayor importancia recae de nuevo en la calidad, ya que el 80 \% de los participantes valora la calidad como un aspecto "muy importante". Otros aspectos que también se consideran de importancia son el precio y la honestidad con un $60 \%$ de puntuación "muy importante", seguidos de la rapidez y la relación entre el cliente y el proveedor, ambos aspectos con un $50 \%$ de puntuación "muy importante". En este caso, aunque el aspecto de los procesos que se han de llevar a cabo en cualquier proyecto de traducción o localización vuelve a ser el valorado como menos importante, este perfil de traductores ha valorado de manera algo más alta la importancia de este aspecto, con un $40 \%$ de puntuaciones como "muy importante".

En cuanto al uso de herramientas informáticas específicas para el control de calidad, se observa de nuevo que una mayoría de traductores que trabajan en empresas o agencias de traducción sí las utilizan (70\%). Como ocurría con el perfil de traductor autónomo, en este caso vemos que la opción más utilizada por aquellos que contestaron positivamente sobre el uso de herramientas informáticas específicas para el control de calidad es el uso de las herramientas integradas en los programas TAO. Sin embargo, no suele ser la única opción de estos participantes, ya que 5 de los 7 participantes que utilizan este tipo de herramientas combinan el uso de las integradas en programas TAO con XBench e, incluso, 3 de estos 5 añaden QA Distiller. Cabe destacar de nuevo que aquellos que han dado respuesta negativa al uso de herramientas informáticas específicas para el control de calidad 
destacan la revisión humana llevada a cabo por otros profesionales de la traducción como medio de control de calidad.

Finalmente, en el bloque de preguntas referente a la posesión de certificaciones de calidad, el $40 \%$ afirma poseer algún tipo de certifiicación, frente al $60 \%$ que no cuenta con ningún certificado en su lugar de trabajo. En este caso, los certificados de calidad mencionados son los siguientes: ISO 17100 (30\%), ISO 900 I $(20 \%)$ y EN I 5038 (I0 \%). En cuanto a la pregunta a aquellos que no poseen ningún certificado de calidad en la actualidad, el $83,3 \%$ ha respondido de manera positiva, planteándose obtener algún tipo de certificación en el futuro.

\subsection{Cliente de servicios de traducción}

Este perfil se ha incluido en el presente estudio para contrastar la opinión y el punto de vista acerca de la calidad que tienen aquellas personas que hayan contratado en algún momento un servicio de traducción o localización. Hemos de señalar que han participado un total de 9 clientes de servicios de traducción y han respondido únicamente al primer bloque de preguntas dedicado a la evaluación de la calidad en el ámbito de la traducción y la localización.

Así, y como ya ocurrió en las respuestas del perfil de traductor autónomo, la mayoría de las respuestas abiertas están planteadas como una evaluación sobre si la calidad en el ámbito de la traducción es buena o mala desde su punto de vista; solo encontramos una respuesta que trata de definir la calidad como concepto: "Que la traducción sea exacta y no varíe el significado del original".

En cuanto a la pregunta sobre los problemas a los que se tiene que enfrentar la calidad en traducción y localización hoy en día, las respuestas de este perfil consideran como problema más importante los requisitos de calidad cada vez más exigente, ya que el $66,6 \%$ lo puntúa como "muy importante" y el $22,2 \%$ como "importante". En un punto intermedio encontramos la problemática de los requisitos de certificación, a la cual un $66,6 \%$ le da una puntuación de "muy importante". Finalmente, el problema al que puntúan como menos importante es la degradación de la calidad, que el $33,3 \%$ de los participantes de este perfil considera "muy importante" y el 55,5\% como "importante".

Por último, en el bloque de evaluación de la importancia de diferentes aspectos durante el proceso de traducción o localización, la mayor puntuación la recibe la honestidad con un $77,7 \%$ de puntuaciones como "muy importante", seguido de un $22,2 \%$ que la considera "importante". En segundo lugar, encontramos la calidad con otro $77,7 \%$ de puntuación "muy importante", pero solo un II,I \% de puntuación "importante". La relación con el cliente o proveedor es el siguiente aspecto mejor valorado, con un $66,6 \%$ de puntuación "muy importante". Seguidamente encontramos el precio, con un $55,5 \%$ de puntuaciones como "muy 
importante". En último lugar en la escala de importancia encontramos la rapidez y los procesos que se han de llevar a cabo en cualquier proyecto de traducción o localización, con un 44,4 \% de puntuación "muy importante" en ambos casos.

\section{Discusión y conclusión}

Atendiendo al primer objetivo de este trabajo, "observar si existen diferencias de uso de las herramientas de control de calidad desde la perspectiva del traductor autónomo, así como desde la perspectiva de la empresa especializada o agencia de traducción", y tal y como se ha podido constatar gracias a los resultados del cuestionario, el uso de estas herramientas está muy extendido entre los traductores. Sin embargo, son los trabajadores en plantilla, ya sea en agencias o en empresas especializadas, los que más las utilizan (lo cual tal vez ocurre debido al alto precio de estas herramientas, fácilmente asumible por una empresa, pero algo más difícil para un autónomo); puede ser por ese motivo que estos últimos prefieran las herramientas integradas en programas de TAO como SDL Trados, Memo Q o Déjà Vu, por ejemplo.

En cuanto a las normas de calidad, el objetivo era evaluar la situación actual de aplicación y cumplimiento de las normas para el control de la calidad, ISO y UNE, en el ejercicio de la traducción y su posible evolución futura. Sin embargo, observamos que su aplicación no está del todo consolidada en este sector. Sobre todo, llama la atención que ninguno de los traductores autónomos participantes en este estudio posea alguna norma de regulación de la calidad. Esto puede deberse al coste de adquisición de estas y porque, tal vez, a nivel autónomo, sin una gran compañía detrás y por el desconocimiento de estas normas por parte de los clientes, no compensa realizar un desembolso y un esfuerzo así. Desde el punto de vista de las empresas o agencias, podríamos pensar que la posesión de alguna norma que asegure la calidad de sus servicios y productos sería un punto a favor para destacarse y diferenciarse frente a los competidores, pero parece no ser el caso ya que menos de la mitad de los traductores en plantilla afirmó poseer uno de estos certificados en su lugar de trabajo. No obstante, cuando se les preguntó sobre la posible adquisición de certificados de calidad en el futuro, la mayoría contestó afirmativamente: esto constata el creciente interés de las empresas de traducción y localización, así como de los traductores que ejercen por cuenta propia, por ofrecer servicios y productos de calidad. A través de estas certificaciones de calidad, estas empresas y traductores autónomos podrían 
asegurar ya no solo que sus traducciones son de calidad, sino que los procesos que llevan a cabo hasta llegar al resultado final también lo son.

Por otro lado, no se ha podido establecer ninguna conexión entre el uso de las herramientas informáticas de control y evaluación de la calidad y la aplicación de las normas anteriormente citadas ya que, como se ha mencionado anteriormente, la mayoría de los traductores autónomos participantes en el estudio hacen uso de las herramientas informáticas de control de calidad, pero no poseen ninguna certificación. En el caso de aquellos que trabajan en una empresa o agencia de traducción tampoco se ha podido establecer conexión ya que la tendencia es parecida; la mayoría hace uso de las herramientas informáticas, pero pocos afirman poseer certificaciones de calidad.

Otro de los objetivos de este estudio era representar la diferencia en la percepción y contrastar la importancia de la calidad desde el punto de vista del traductor profesional, ya sea autónomo o trabajador en plantilla, así como desde la perspectiva del cliente, para observar las posibles diferencias o distorsiones en su percepción. Para esto se les preguntó a los participantes de los tres perfiles que definieran la calidad en el ámbito de la traducción y la localización desde su punto de vista. Lo primero que nos llama la atención es la interpretación de la pregunta por parte de muchos participantes, sin importar el perfil, que han entendido la pregunta como una valoración del estado de la calidad en los productos ya traducidos o localizados actualmente. Si nos centramos en las respuestas que ofrecen una definición de la calidad en traducción y localización, observamos que la mayoría de estas se centran en aspectos como la corrección y la naturalidad, sin importar el perfil del participante. Estos dos son los pilares que definen la calidad ya que un producto plagado de faltas y errores, por muy naturales que sean las expresiones o códigos utilizados para la cultura meta, no resultará en un producto de calidad.

En cuanto a la identificación de problemas relacionados con la calidad, hemos observado diferencias en las opiniones de los tres perfiles, ya que para los traductores autónomos o freelance el mayor problema son los requisitos de calidad cada vez más exigentes. Este factor puede ocasionar problemas de entendimiento con el cliente, ya que a mayor calidad, mayor precio del servicio a causa de la dificultad de los procesos y el tiempo que se ha de invertir en ellos. Sin embargo, desde el punto de vista del perfil de los clientes, el mayor problema también son los requisitos de calidad cada vez más exigentes, por lo que podríamos afirmar que en este ámbito los clientes potenciales están entendiendo progresivamente que "la calidad se paga" y son conscientes de que la traducción y la localización son procesos costosos de realizar. Por otro lado, desde el punto de vista de los trabajadores en empresas o agencias de traducción el mayor problema es la degradación de la calidad. Esto puede deberse a la presión que reciben al trabajar 
en una empresa o agencia donde no tienen contacto directo con el cliente, de manera que reciben el encargo a través de un superior, en ocasiones por partes si se trata de un encargo de gran volumen, lo que puede ocasionar incoherencias en cuanto a los criterios de calidad y, por lo tanto, su degradación.

La calidad es un aspecto que se considera de gran importancia en el ámbito de la traducción y la localización desde el punto de vista de los profesionales de este sector, tanto autónomos como trabajadores en empresas o agencias de traducción; desde el punto de vista de los clientes, no obstante, se le da más importancia a la ética y la honestidad en la relación proveedor-cliente, seguido de la calidad. Teniendo estos dos aspectos en cuenta, podemos asegurar que la comunicación entre el profesional de la traducción y el cliente es fundamental, establecer unos parámetros claros que aseguren la máxima calidad en el servicio, o el nivel de calidad exigido por el cliente, es la base para que el producto resultante sea óptimo. Todo esto debería unirse al cumplimiento de unas normas internacionales que aseguren la calidad tanto de los procesos como del servicio ofrecido o del producto final.

Finalmente, con la reciente actualización de la norma internacional UNE-EN ISO 17100:2015, puesta en marcha desde noviembre de 2015 (AENOR, 2016), sería interesante retomar un estudio como este dentro de unos años para ver si alguno de los parámetros u opiniones ha cambiado y, sobre todo, si el estado de la calidad en el ámbito de la traducción y la localización ha mejorado.

\section{Referencias bibliográficas}

AEC. (2016). Aseguramiento de la calidad. Recuperado de: http://www.aec.es/web/guest/centro-conocimiento/aseguramiento-de-lacalidad.

AENOR. (20I6). Certificación de la Calidad. Recuperado de: http://www.aenor.es/.

AREVAlillo, J. J. (2004). "A propósito de la norma europea de calidad para los servicios de traducción”. Las palabras del traductor. Actas del II Congreso «El español, lengua de traducción» 20 y 21 de mayo, 2004 Toledo. 89-100. Recuperado http://cvc.cervantes.es/lengua/esletra/pdf/02/013 arevalillo.pdf.

(2006). "La norma europea de calidad para servicios de traducción EN15038: por fin, una realidad”. Panace@. Revista de Medicina, Lenguaje y Traducción, 7(23), 107-III.

Hatim, B., y MASON I. (1995). Teoría de La Traducción: Una Aproximación Al Discurso. Madrid: Ariel.

ISO. (2016). About ISO. Recuperado de: http://www.iso.org/iso/home/about.htm. Recuperado el 5 de febrero de 2016. 
KeEney, S., Hasson, F., y MCKenNA, H. P. (200I). A critical review of the Delphi technique as a research methodology for nursing. International Journal of Nursing Studies, 38(2), 195-200.

Koby, G., Fields, P., Hague, D., Lommel, A., y Melby, A. (20/4). “Defining translation Quality”. Revista TradumàTica: Tecnologies De La Traducció, 0( I2), 4I3-420.

Recuperado de http://revistes.uab.cat/tradumatica/article/view/n I2-koby-fields-hagueetal. Recuperado el 5 de febrero de 2016.

MACKEY, A., y GASS, S. M. (2005). Second language research: Methodology and design. Mahwah, NJ: Lawrence Erlbaum Associates.

MARTíNEZ, R. (20I4). "A deeper look into metrics for translation quality assessment (TQA): a case study”. Miscelánea: a journal of English and American studies, 49, 73-94.

PyM, A. The Moving Text: Localization, Translation, and Distribution. (2004). Benjamins Translation Library. John Benjamins Publishing Company, 2004. Recuperado de: https://books.google.es/books?id=kVU9AAAAQBAJ.

Real Academia Española de la Lengua (RAE). (20I6). Diccionario de la Lengua Española. Recuperado de www.rae.es .

SCHÜTZ, Jorg. (1999). "Deploying the SAE J2450 Translation Quality Metric in Language Technology Evaluation Projects". Proceedings of the 2 Ist ASLIB Conference Translating and the Computer, London, Great Britain. I0-II November 1999. Recuperado de: http://mt-archive.info/Aslib-1999Schutz.pdf.

Wright, K. B. (2006). Researching Internet-Based Populations: Advantages and Disadvantages of Online Survey Research, Online Questionnaire Authoring Software Packages, and Web Survey Services. Journal of Computer-Mediated Communication, 10(3). Recuperado de http://onlinelibrary.wiley.com/doi/I0. I I I I/j.1083-6 I0I.2005.tb00259.x/full 
